\title{
Local Drug Delivery Strategy for Cancer Treatment: Use of Biocompatible Sol-Gel-Derived Porous Materials
}

\author{
Odile Cristini-Robbe ${ }^{1}$, Florian Ruyffelaere ${ }^{2}$, Florent Dubart ${ }^{2}$, Ange Uwimanimpaye ${ }^{2}$, \\ Christophe Kinowski $^{1}$, Remy Bernard ${ }^{1}$, Catherine Robbe-Masselot ${ }^{3}$, Ikram El Yazidi $^{3}$, Sylvia Turrell ${ }^{2}$ \\ ${ }^{1}$ PhLAM (UMR 8523), Université Lille1, Villeneuve d'Ascq, France; ${ }^{2}$ LASIR (UMR 8516), Université Lille1, Villeneuve d'Ascq, \\ France; ${ }^{3}$ Unité Glycobiologie (UMR 8576), Université Lille1, Villeneuve d'Ascq, France. \\ Email: odile.robbe@univ-lille1.fr
}

Received November $10^{\text {th }}, 2012$; revised December $10^{\text {th }}, 2012$; accepted December $23^{\text {rd }}, 2012$

Copyright (C) 2013 O. Cristini-Robbe et al. This is an open access article distributed under the Creative Commons Attribution License, which permits unrestricted use, distribution, and reproduction in any medium, provided the original work is properly cited.

\begin{abstract}
Porous silica xerogel materials have been developed to use as drug-release agents to be implanted directly in or near cancerous tissues. In order to test the capacity of the materials to absorb and then to release medicinal substances, a battery of examinations (UV and visible micro-Raman, porosity measurements, UV-visible absorption spectra) have been made using test drug molecules (clotrimazole, primaquine diphosphate and the anti-cancer agent vinblastine sulphate). Results show that the molecules can be post-doped into the gels and the Raman data provide indications of the best conditions for detecting the substances absorbed in the gels. Spectroscopic results show that the drug molecules are released by the xerogel over a period of 10 days. These results are promising for the development of these materials as drug-release agents.
\end{abstract}

Keywords: Sol-Gel; Micro-Raman; Post-Doping; Chemotherapy; Silica Gels; Drug-Release Agents

\section{Introduction}

The most common combinational strategies for effective cancer treatment generally involve the combination of multiple chemotherapeutic agents, chemotherapy with radiotherapy, etc. $[1,2]$. However, one of the biggest problems in cancer therapy is that of dose-dependent toxicity toward the non-cancerous cells, since poor drug accessibility to the tumor site necessitates high doses of drug treatment [1]. Hence, in order to improve overall survival and quality of life, it is now desired to increase the bioavailability of drugs to the disease sites and to contain the delivery to the cancerous tissues.

Existing systems for this approach rely either on 1systemic delivery (polymer nanoparticle systems, for example, which are administered intravenously but which have difficulty being well localized because of sequestration by the reticulendothelial system [2,3]) or 2controlled release drug delivery depot systems for implantation intra-tumorally or adjacent to cancerous tissue [3]. Though such tested materials are tolerated well in vivo, they generally have poor solubility in organic solvents thus restricting the inclusion of water-insoluble chemotherapy agents.
Previous reports using porous silica substrates have all been sol-doped systems - thus requiring water soluble additives [4-9]. In order to circumvent these problems, we propose an approach previously unreported, that of post-doping biocompatible silica gels [10] with the adequate chemotherapy agent. With this in mind, we have begun tests first to determine the capacity of the gels to absorb the molecular agents and then, in a second step, to test the ability of the gels to release their absorbed molecular agents to the environment.

\section{Sample Preparation}

Mesoporous silica matrices were obtained using the ammonium hydroxide catalyzed sol-gel process of hydrolysis and condensation of tetramethyl orthosilicate (TMOS, $\leq 99 \%$ ) at $30^{\circ} \mathrm{C}$, under atmospheric pressure [11]. The samples obtained were heat-treated first at $120^{\circ} \mathrm{C}$ and then at $850^{\circ} \mathrm{C}$ in air to eliminate all residual organic species and the major part of $\mathrm{Si}-\mathrm{OH}$, to exclude water from the pores and also to stabilize the resulting xerogels. The resulting monoliths were glassy, colorless, and crack-free. These xerogels were soaked for $12 \mathrm{~h}$ in aqueous solutions of test molecules (clotrimazole and the photoluminescent 
primaquine diphosphate) and of the anticancer agent vinblastine sulphate. The doping solutions consisted of 100 $\mathrm{mg}$ of the test drug molecule in $5 \mathrm{~mL}$ of distilled water. Accordingly, each sample contained about $12.3 \mathrm{mg}$ of drug per gram of $\mathrm{SiO}_{2}$ gel. In the case of vinblastine sulphate, $10 \mathrm{mg}$ were dissolved in $5 \mathrm{~mL}$ of water. The gels were then dried at $50^{\circ} \mathrm{C}$ for $24 \mathrm{~h}$. All precursors and solvents were purchased from Aldrich and all compounds were used without further purification.

Silica, clotrimazole, primaquine diphosphate, vinblastine sulphate, clotrimazole/silica, primaquine diphosphate/silica and vinblastine sulphate/silica will be hereafter denoted as $\mathrm{SiO}_{2}, \mathrm{Cl}, \mathrm{Pr}, \mathrm{Vi}, \mathrm{Cl}-\mathrm{Si}, \mathrm{Pr}-\mathrm{Si}$ and $\mathrm{Vi}-\mathrm{Si}$, respectively.

\section{Experimental Techniques}

In order to determine the porosities of the silica gels before and after dopage, adsorption-desorption isotherms were obtained using a BET Quantachrome Autosorb-1 system.

Raman spectra of undoped and doped gels were recorded with a Horiba-Jobin Yvon Labram HR spectrometer excited with the $632.8,532$ and $473 \mathrm{~nm}$ lines of a He-Ne laser and with a Horiba-Jobin Yvon visible UV micro Raman spectrometer excited with the 266 and 325 $\mathrm{nm}$ lines of a HeCd laser. In order to follow the amount of dopant molecules released into solution, UV-visible spectra were measured with a double-beam Cary 5000 instrument.

\section{Results and Discussion}

\subsection{Characterization of Drug Molecules and Carrier Matrices}

The chemical structures of the tested drugs ( $\mathrm{Cl}, \mathrm{Pr}$ and $\mathrm{Vi})$ are presented in Figure 1. Photographs of an undoped porous silica xerogel and a Pr-doped silica matrix are shown in Figure 2(a). The cylindrical xerogels obtained after doping with drug molecules remained unbroken and without visual inhomogeneities.

Two important aspects in the design and testing of a delivery system is whether or not the drug molecule can be detected in the carrier matrix and whether or not there is interaction between the two. In the present work, the gels were first examined to determine the best method of detecting the absorbed species. The assignments of the Raman bands observed for $\mathrm{SiO}_{2}$ xerogel, in Figure 2(b), are presented in Table 1 . Of these bands, those around 430,490 and $600 \mathrm{~cm}^{-1}$ assigned to the network Si-O-Si bending vibration, and to the symmetric stretching modes of Si-O bonds in 4- and 3-membered silica rings, respectively, are the most important signals of the silica matrix. In the micro-Raman spectra of Figure 2(c), test results obtained using primaquine diphosphate before (A) and

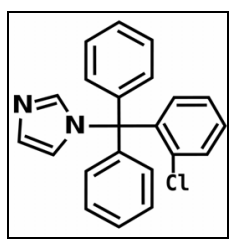

(a)

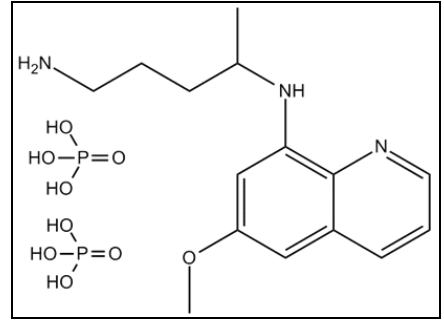

(b)

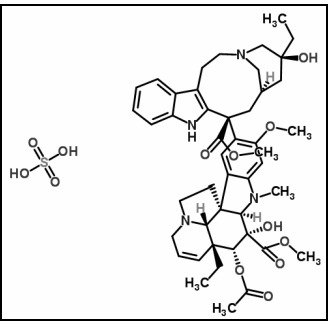

(c)
Figure 1. Structural formulas of (a) clotrimazole, (b) primaquine diphosphate and (c) vinblastine sulphate.

after (B) doping are presented. These spectra, obtained with an excitation at $632.8 \mathrm{~nm}$, show the fluorescence interference often encountered with colored samples. However when excited with UV excitation at $266 \mathrm{~nm}$, the spectrum (C) shows a fluorescence-free signal, which unfortunately can be assigned uniquely to carbon thus demonstrating that the high laser energy resulted in a calcination of the samples. Using the characteristic vibrational frequencies for Pr which are presented in Table 2, it can be seen that in spite of the fluorescence, all the bands characteristic of Pr are detected in the doped gel (spectrum B of Figure 2(b)). In the region of $463 \mathrm{~cm}^{-1}$, one can see the contribution of the Si-O-Si matrix. The fact that no frequency changes are observed between $\mathrm{A}$ and $\mathrm{B}$, indicates that there is no interaction between $\mathrm{Pr}$ and $\mathrm{SiO}_{2}$. As the molecule is not affected by the matrix, when it is released, it will still be in its normal active form.

In a similar way, the spectrum of Cl-Si, in Figure 2(d), shows bands characteristic of $\mathrm{Cl}$ (See Table 3: 1008, 1161 and $1460 \mathrm{~cm}^{-1}$ ). In this case, as the drug is colorless, there is no problem with fluorescence and the spectrum of the gel is clearly obvious (430, 492 and $815 \mathrm{~cm}^{-1}$ ). Similarly, Vi could be identified in the spectra of $\mathrm{Vi}-\mathrm{Si}$ samples.

These data show that micro-Raman spectroscopic investigation of doped silica matrices can detect the presence of doping species and can also indicate whether or not there is an interaction between the carrier matrix and the drug molecule. Obviously, an interaction would imply a structural modification of the drug molecule and hence a possible change in its mechanism of interaction.

The porosity of the drug-incorporated matrix also plays an important role in release kinetics. For determination of the texture (porous volume, pore size distribu- 


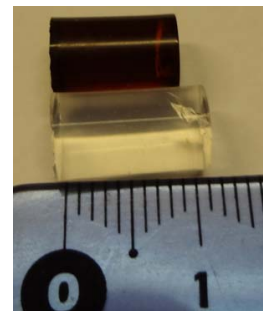

(a)

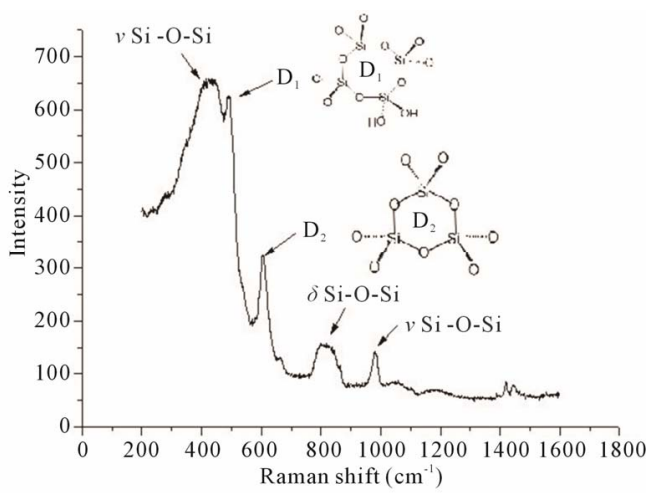

(b)

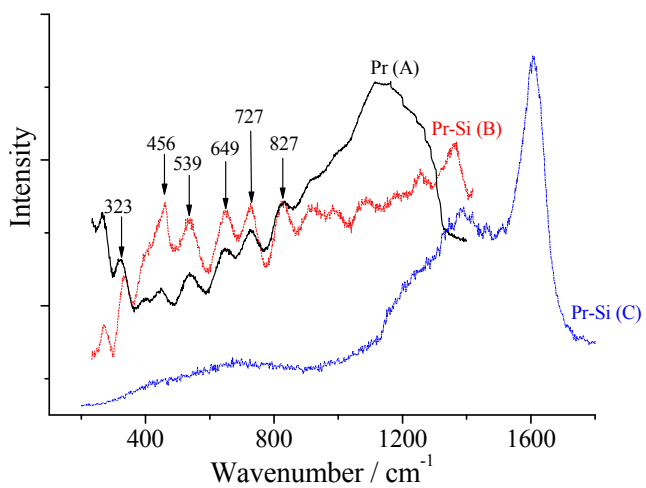

(c)

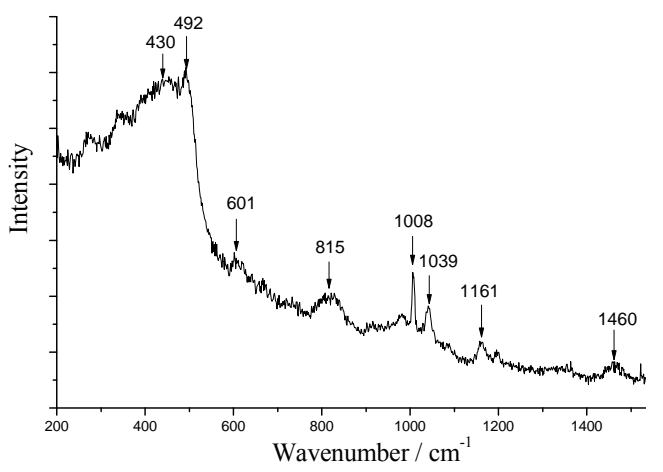

(d)

Figure 2. (a) Photograph of undoped (transparent, $\mathrm{SiO}_{2}$ ) and primaquine-doped (red, Pr-Si) silica xerogel matrices; (b) Raman spectrum of undoped $\mathrm{SiO}_{2}$ obtained with a 632.8 nm excitation; (c) Raman spectra of Pr (A) and Pr-Si (B) obtained with a $632.8 \mathrm{~nm}$ excitation. Pr-Si (C) is obtained with a $266 \mathrm{~nm}$ excitation; (d) Raman spectrum of Cl-Si with a $632.8 \mathrm{~nm}$ excitation.
Table 1. Assignments of Raman bands of silica xerogel.

\begin{tabular}{cc}
\hline Wavenumber $/ \mathrm{cm}^{-1}$ & Attribution \\
\hline 430 & $v$ Si-O-Si (TOT band) \\
490 & $\mathrm{D} 1$ \\
600 & $\mathrm{D} 2$ \\
800 & $\delta \mathrm{Si-O}-\mathrm{Si}$ \\
980 & $v$ Si-OH \\
\hline
\end{tabular}

Table 2. Assignments of Raman bands of primaquine diphosphate powder.

\begin{tabular}{cc}
\hline Wavenumber $/ \mathrm{cm}^{-1}$ & Attribution \\
\hline 265 & $\mathrm{C}-\mathrm{N}-\mathrm{C}, \mathrm{C}-\mathrm{CH}_{3}$ out of plane \\
323 & $\mathrm{C}-\mathrm{C}-\mathrm{C}$ \\
449 & $\delta$ out of plane N-C-C \\
539 & $\delta$ out of plane C-C-C \\
646 & $\delta$ out of plane, $\omega \mathrm{NH}_{2}$ and $\mathrm{CH}_{3}$ \\
727 & $\omega \mathrm{NH}_{2}$ and $\mathrm{CH}_{3}$ \\
827 & $\mathrm{C}-\mathrm{C}-\mathrm{C}$ \\
$950-1400$ & Phosphate groups \\
\hline
\end{tabular}

Table 3. Assignments of Raman bands of clotrimazole powder.

\begin{tabular}{cc}
\hline Wavenumber $/ \mathrm{cm}^{-1}$ & Attribution \\
\hline 1004 & $v \mathrm{~N}-\mathrm{C}$ \\
1160 & $\delta \mathrm{CH} / \mathrm{NH}$ \\
1460 & $\delta \mathrm{CH} / \mathrm{CH}_{2}$ of rings \\
1600 & $\delta$ phenyl \\
\hline
\end{tabular}

tion and pore interconnection), the nitrogen adsorptiondesorption technique was used. The shape of the adsorption-desorption isotherms and the type of hysteresis loops were described using the Brunauer et al. model and the de Boer correlations, respectively $[12,13]$. The specific surface areas were determined using the Brunauer-Emmett-Teller (BET) method [14]. The pore-size distributions were thus given by the Barett-Joyner-Hal-enda (BJH) formula which determines the derivative of the desorbed volume as a function of the pore diameter [15]. For the following sections, only results concerning primaquine diphosphate will be presented.

As can be seen in Figure 3(a), the isotherms for undoped and Pr-doped xerogels show reversible sections at low pressures and hysteresis loops at higher pressures. These loops of the type IV isotherms are characteristic of mesoporous samples with interconnected pores. Com- 


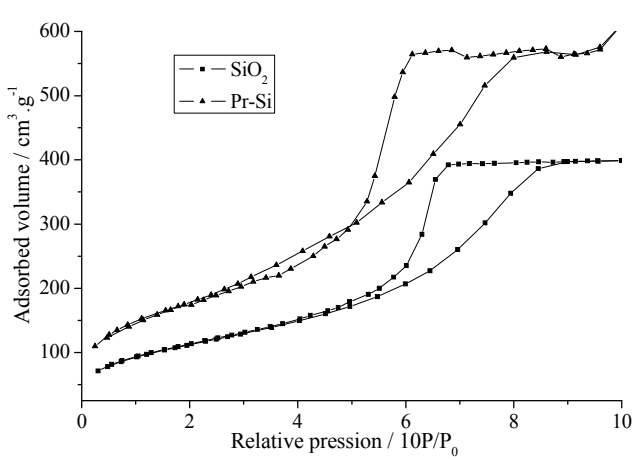

(a)

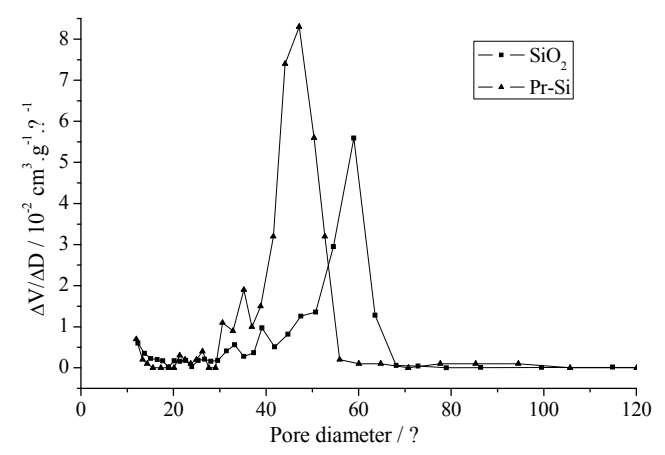

(b)

Figure 3. (a) Nitrogen adsorption-desorption isotherms of $\mathrm{SiO}_{2}$ and Pr-Si; (b) BJH desorption pore-size distributions of $\mathrm{SiO}_{2}$ and $\mathrm{Pr}-\mathrm{Si}$.

parison of the two curves shows an increase in total pore volume after doping with $\operatorname{Pr}$ (from $0.62\left(\mathrm{SiO}_{2}\right)$ to 0.94 $\left.\mathrm{cm}^{3} \cdot \mathrm{g}^{-1}(\mathrm{Pr}-\mathrm{Si})\right)$. The corresponding specific surface area increases from 375 to $671 \mathrm{~m}^{2} \cdot \mathrm{g}^{-1}$. Determination of the pore diameters shows that the mean pore diameter (Figure 3(b)) decreases with doping, from $6 \mathrm{~nm}$ for $\mathrm{SiO}_{2}$ to $4.7 \mathrm{~nm}$ for Pr-Si.

These results demonstrate that the doping has a marked effect on the porous structure of this carrier matrix. Remembering that these materials are porous gels and not glasses, the data suggest that during post-doping, the gel behaves like a sponge, with inflation of pore volume and specific surface. The apparent decrease in pore size after doping could be due to the occupation of space within the pores by the primaquine diphosphate molecules, which would limit access of the infiltrating nitrogen gas.

Another important aspect of these results is that these porous gels can be adapted to the use with a multitude of drug molecules. For, as the carrier pores apparently expand during soaking procedures, it can be deduced that the size of the matrix pores does not pose rigid limitations on the size of the molecules to be inserted.

\subsection{Release Properties}

Release properties of the $\mathrm{SiO}_{2}$ matrix were studied by soaking the Pr-doped gels in distilled water in order to test the discharge of the drug molecules into the surrounding liquid. In order to quantify the amount of drug eluted, UV-visible absorption spectra were first measured for standard solutions of primaquine diphosphate in order to establish a Beer-Lambert curve (See Figure 4(a)).

UV-visible absorption spectra were then measured of the liquid used for soaking the samples at various times up to 10 days of elution (Figure 4(b)). The drug release pattern for Pr-Si, presented in Figure 4(c), shows a biphasic behavior. There is an initial "burst" of $3.5 \mathrm{mg}$ per gram of $\mathrm{SiO}_{2}$ gel, released on the first day. The amount of primaquine diphosphate molecules diffused from the gel decreases to $0.40 \mathrm{mg}$ per gram of $\mathrm{SiO}_{2}$ gel after 4 days. A sustained diffusion of this amount of primaquine continues up to 10 days.

As the samples were consistently washed before elution measurements, all molecules released were issue of the inner pores of the xerogel, not from its surface. The decreased rate of diffusion of the drug from the matrix with time might be due to the lesser driving force for diffusion which is proportional to the availability of entrapped drug molecules.

In general, in the case of an insoluble porous carrier, in which the release is governed by diffusion of the drug from the matrix, release kinetics follows a conventional Higuchi relationship (variation of drug vs. square root of time). Accordingly, the plot of Figure 4(d) shows a two-step release profile. Since our Raman studies have shown that there are no chemical interactions between the drug and the matrix, this behavior must be attributed to physical entrapping and the elution is, in fact, therefore diffusion controlled.

These release properties show that a porous silica matrix can be effectively post-doped by immersion in a drug solution. Moreover, the resulting systems act as effective carriers for drug delivery making it possible to maintain a sustained release of the drug molecules.

\section{Conclusions}

The present work shows that not only is sol-gel processing a practical technique for the fabrication of carrier substrates, but that post-doping procedures are easily adaptable for the conception of loaded drug delivery materials. These silica xerogels post-doped with aqueous solutions of several drug molecules have been studied by Raman and UV-visible absorption spectroscopies and nitrogen adsorption-desorption techniques.

Nitrogen adsorption-desorption isotherm studies have shown that the present biocompatible silica materials have high surface areas and sufficiently large pore-sizes, making them good for post-doping. BET measurements show that soaking the gels in drug solutions causes an increase of the total pore volume and the specific surface 


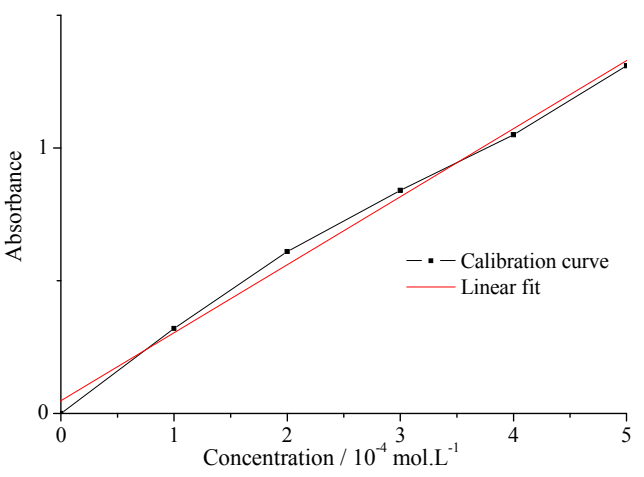

(a)

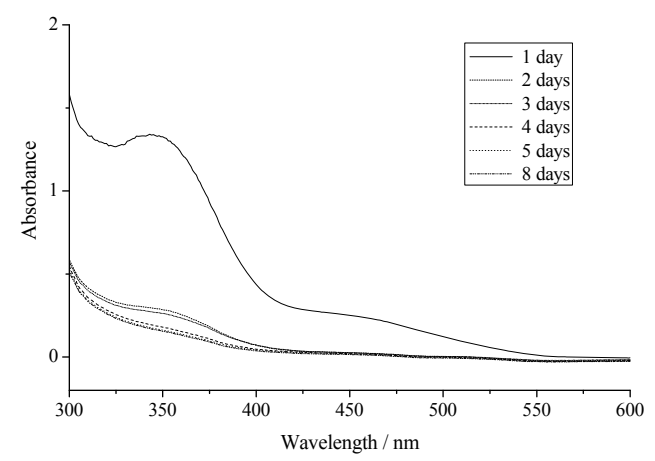

(b)

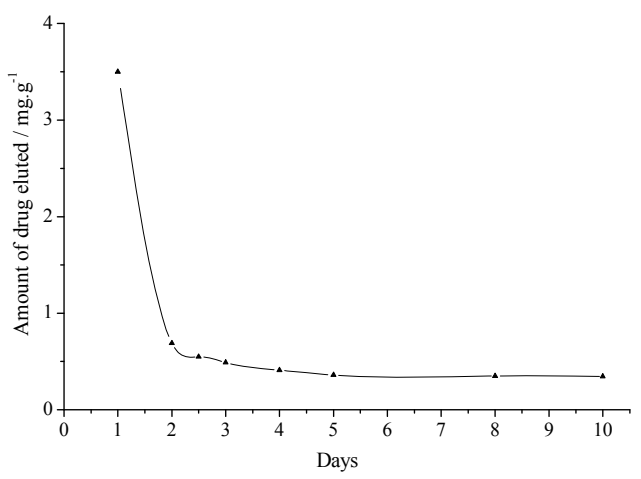

(c)

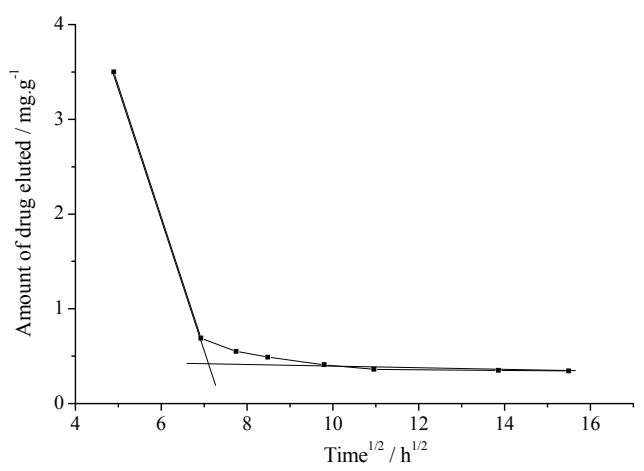

(d)

Figure 4. (a) Beer-Lambert calibration curve for primaquine diphosphate aqueous solutions; (b) UV-visible absorption spectra obtained for drug-eluted solutions at various times; (c) Elution patterns of Pr-Si gel; (d) Amount of drug eluted vs. the square root of time for Pr-Si. area. Raman spectra show that after post-doping the drug molecules are indeed entrapped within the silica matrix, but with no apparent chemical interactions between the two.

Use of the Beer-Lambert curve for Primaquine diphosphate solutions and the UV-visible spectra of the solutions used for soaking the Pr-Si samples made it possible to quantify the amount of Pr released from the loaded gels. Results show that drug release begins with an initial value of $3.5 \mathrm{mg}$ per gram of $\mathrm{SiO}_{2}$ gel. The elution decreases to a sustained value of $0.40 \mathrm{mg}$ per gram of $\mathrm{SiO}_{2}$ gel for at least 10 days.

These studies point to the possibility of developing these systems for implantable drug-release agents.

\section{REFERENCES}

[1] P. Parhi, C. Mohanty and S. Sahoo, "NanotechnologyBased Combinational Drug Delivery: An Emerging Approach for Cancer Therapy," Drug Discovery Today, Vol. 42, No. 3, 2001, pp. 291-293.

[2] S. Prakash, M. Malhotra, W. Shao, C. Tomaro-Duchesneau and S. Abbasi, "Polymeric Nanohybrids and Funtionalized Carbon Nanotubes as Drug Delivery Carriers for Cancer Therapy," Advanced Drug Delivery Reviews, Vol. 63, No. 14-15, 2011, pp. 1340-1351. doi:10.1016/j.addr.2011.06.013

[3] J. Wolinshky, Y. Colson and M. Grinstaff, "Local Drug Delivery Strategies for Cancer Treatment: Gels Nanoparticles, Polymeric Films, Rods and Wafers," Journal of Controlled Release, Vol. 159, No. 1, 2012, pp. 14-26. doi:10.1016/i.jconrel.2011.11.031

[4] P. P. Nampi, V. S. Mohan, A. K. Sinha and H. Varma, "High Surface Area Sol-Gel Nano Silica as a Novel Drug Carrier Substrate for Sustained Drug Release," Materials Research Bulletin, Vol. 47, No. 1, 2012, pp. 1379-1384. doi:10.1016/j.materresbull.2012.03.003

[5] V. Ambrogi, L. Perioli, C. Pagano, F. Marmottini and M. Ricci, "Use of SBA-15 for Furosemide Oral Delivery Enhancement," European Journal of Pharmaceutical Sciences, Vol. 46, No. 1-2 , 2012, pp. 43-48. doi:10.1016/j.ejps.2012.02.004

[6] R. Chen, H. Qu, A. Agrawal, S. Guo and P. Ducheyne, "Controlled Release of Small Molecules from Silica Xerogel with Limited Nanoporosity," Journal of Materials Science Materials in Medicine, Vol. 24, No. 1, 2012, pp. 1-10. doi:10.1007/s10856-012-4783-3

[7] S. Wang, "Ordered Mesoporous Materials for Drug Delivery," Microporous and Mesoporous Materials, Vol. 117, No. 1-2, 2009, pp. 1-9. doi:10.1016/j.micromeso.2008.07.002

[8] G. Maria, D. Berger, S. Nastase and I. Luta, "Kinetic Studies on the Irinotecan Release Based on Structural Properties of Functionalized Mesoporous-Silica Supports," Microporous and Mesoporous Materials, Vol. 149, No. 1, 2012, pp. 25-35.

doi:10.1016/j.micromeso.2011.09.005 
[9] K. Ariga, A. Vinu, J. P. Hill and T. Mori, "Coordination Chemistry and Supramolecular Chemistry in Mesoporous Nanospace," Coordination Chemistry Reviews, Vol. 251, No. 21-24, 2007, pp. 2562-2591. doi:10.1016/j.ccr.2007.02.024

[10] B. Menaa, F. Menaa, C. Aiolfi-Guimaraes and O. Sharts, "Silica Based Nanoporous Sol-Gel Glasses: From Bioencapsulation to Protein Folding Studies," International Journal of Nanotechnology, Vol. 7, No. 1, 2010, pp. 1-45. doi:10.1504/IJNT.2010.029546

[11] O. Robbe, K. Woznica, E. Berrier, G. Ehrhart, B. Capoen, M. Bouazaoui and S. Turrell, "Raman Spectroscopic Investigations on the Kinetics of Gelation and Densification of $\mathrm{Cd}^{2+}$ - and $\mathrm{Pb}^{2+}$-Doped Silica Glasses under Basic Conditions," Thin Solid Films, Vol. 515, No. 1, 2006, pp. 73-79. doi:10.1016/j.tsf.2005.12.038
[12] S. Brunauer, L. S. Deming, W. S. Deming and E. Teller, "On a Theory of the Van Der Waals Adsorption of Gases," Journal of the American Chemical Society, Vol. 62, No. 7, 1940, pp. 1723-1732. doi:10.1021/ja01864a025

[13] J. H. De Boer, "The Structure and Properties of Porous Materials," Butterworths, London, 1958.

[14] S. Brunauer, P. H. Emmett and E. Teller, "Adsorption of Gases in Multimolecular Layers," Journal of the American Chemical Society, Vol. 60, No. 2, 1938, pp. 309-319. doi:10.1021/ja01269a023

[15] E. P. Barret, G. L. Joyner and P. P. Halenda, "The Determination of Pore Volume and Area Distributions in Porous Substances. I. Computations from Nitrogen Isotherms," Journal of the American Chemical Society, Vol. 73, No. 1, 1951, pp. 373-380. doi:10.1021/ja01145a126 